\title{
Electrostatically-ACtuated Micro Cantilevers for Electro-Mechanical Measurement of Single-Walled Carbon Nanotubes Grown by Catalytic CVD
}

\author{
Kazunori Hoshino ${ }^{1}$, Yoichi Murakami ${ }^{2}$, Shigeo Maruyama ${ }^{2}$, Kiyoshi Matsumoto ${ }^{1}$ and Isao Shimoyama ${ }^{1}$ \\ ${ }^{1}$ Depertment of Mechano-Informatics, ${ }^{2}$ Department of Mechanical Engineering \\ \{hoshino,matsu, isao\}@leopard.t.u-tokyo.ac.jp, \{murakami, maruyama\}@photon.t.u-tokyo.ac.jp \\ The University of Tokyo, 7-3-1 Hongo, Bunkyo-ku, Tokyo 113-8656, Japan
}

\begin{abstract}
We have build a system for real-time in-situ measurement of electromechanical properties of single-walled carbon nanotubes (SWNTs) growing between electrostatically actuated silicon cantilevers faced each other with a micrometer-order gap. Our catalytic CVD method using ethanol as a carbon source was employed along with a newly developed technique that can synthesize SWNTs directly on the surface of Si substrate at the CVD temperature of as low as $650{ }^{\circ} \mathrm{C}$. SWNTs were successfully grown between the 10 -micrometer gap of the measuring probes. Mechanical properties of the probing cantilevers under the CVD condition were then measured to assess the feasibility of real-time measurement of SWNT growth.
\end{abstract}

\section{INTRODUCTION}

Techniques for arranging carbon nanotubes on MEMS structures have recently become one of the most important topics in NEMS. Although several studies on multi-walled carbon nanotubes (MWNTs) have been reported so far [8], many of the fascinating properties such as metal-semiconducting duality and high transport characteristics are unique to single-walled carbon nanotubes (SWNTs) [2].

In [5], [6] and [7], we proposed a low temperature catalytic CVD process, which used alcohol as a carbon source to reduce the reaction temperature from the typical $900^{\circ} \mathrm{C}$ [1] to as low as $650^{\circ} \mathrm{C}$. The process demonstrated the synthesis of high-quality SWNTs directly on $\mathrm{Si}$ and $\mathrm{SiO}_{2}$ substrates without using any support of under-layer materials. The process's compatibility with the MEMS technology enabled us to take full advantage of the fabrication techniques for silicon micro cantilevers and structures [3].

Post-process measurements of SWNTs using AFM probes were reported by other groups [9]. Our approach is different in trying to explore the in-situ, real-time properties of SWNTs grown under the proposed CVD condition. Ac- cording to the elastic performance of Si microstructures at around $600{ }^{\circ} \mathrm{C}$ [4], it is possible to apply oscillating cantilevers to measure electro mechanical properties of SWNTs under the growing process.

\section{EXPERIMENTAL SETUP}

The SWNTs were synthesized in the following steps. The metal catalyst of Mo and Co were mounted on the test structure by the dip-coat method. The test substrate was dipped into a solution of molybdenum acetate and cobalt acetate. It was then placed in a quartz reaction tube, the inside of which was evacuated under $2 \mathrm{~Pa}$ and heated to the reaction temperature of $600 \sim 900^{\circ} \mathrm{C}$. During the CVD process, ethanol vapor preheated by an electric furnace was introduced to the quartz tube at $1.3 \mathrm{kPa}$. Figure 1 shows SEM examples of SWNTs successfully grown between silicon test structures, the gap between which approaches 10 $\mu \mathrm{m}$ at maximum.

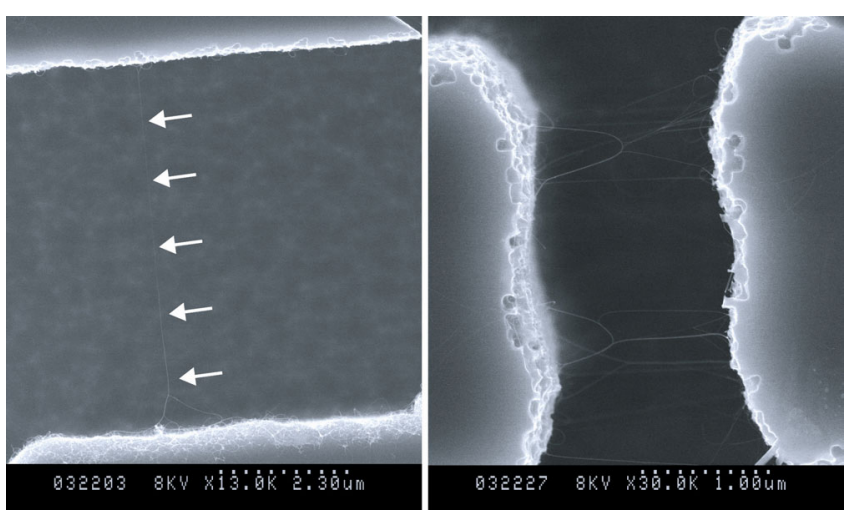

Figure 1. SEM photographs of bridging SWNT bundles on test silicon structures achieved by our catalytic CVD process.

The real-time measurement is based on taking the frequency response of oscillating cantilevers. Changes in the probing cantilever's resonant frequency give the mechanical properties of nanotubes grown on/between the cantilevers. Figure 2 shows the experimental setup to measure the can- 
tilever oscillation under the CVD condition. An electrostatically-actuated cantilever, instead of a conventional piezoelectric actuator, was installed in a quartz reaction tube for the operation under high temperature conditions. The outside diameter of the tube was $30 \mathrm{~mm}$. The cantilever was heated by a spot lamp heater. A 488-nm Ar laser was focused on the oscillating cantilever and the angular displacement of the lever was measured by position sensitive photo-diodes (PSD). For the PSD, band pass filters were used to eliminate the thermal radiation due to the spot heating.

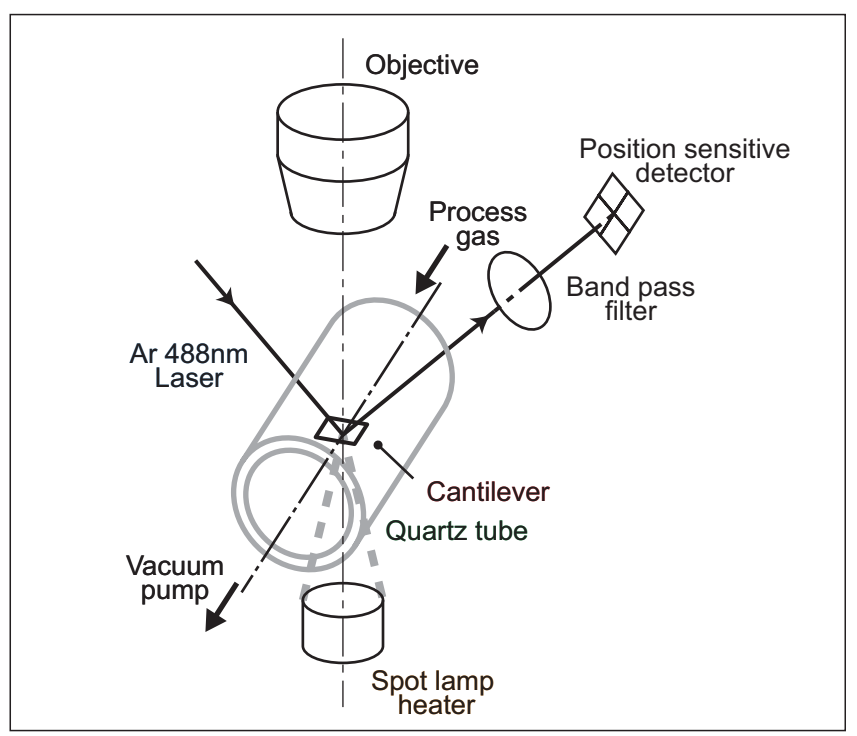

Figure 2. Setup for the real-time measurement of SWNT growth.

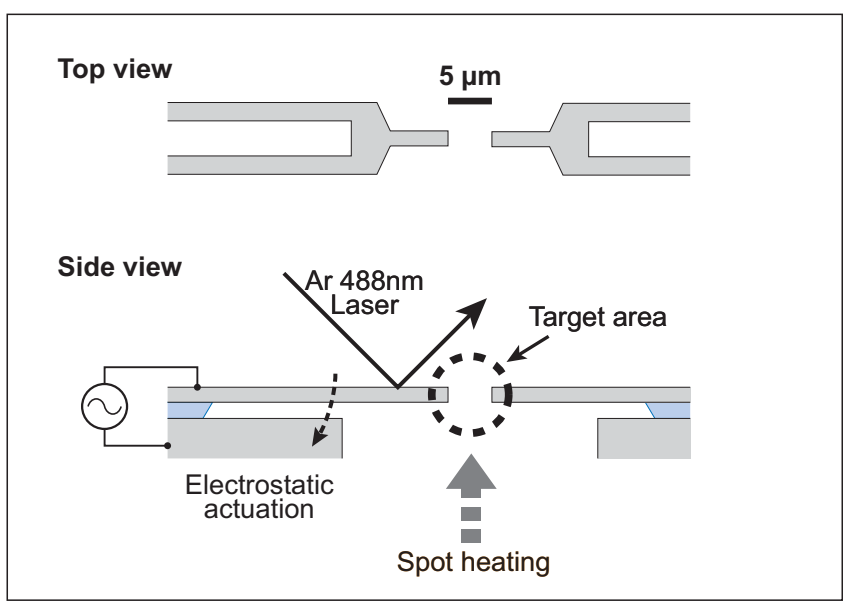

Figure 3. Design of the cantilever pair fabricated on an SOI wafer. One of the pair was actuated. SWNTS were grown between the cantilevers.

The arrangement of the cantilevers is shown in figure 3 (See also figure 4 upper left for an SEM photograph). The cantilever was fabricated on a SOI wafer, which comprises a $2 \sim 3 \mu \mathrm{m}$-thick $\mathrm{Si}$ layer and a $2 \mu \mathrm{m}$-thick $\mathrm{SiO}_{2}$ layer. We etched through the Si substrate to prevent the Ar laser from scattering. The cantilever oscillates by the electrostatic force between the lever and the substrate. The electrodes and wires for the electrical connection were all made of titanium, which does not affect the SWNT synthesis. We have another cantilever on the counter side with the tip-to-tip gap of $5 \mu \mathrm{m}$. Both of the cantilevers have the electrodes which allow electrical measurement of the bridging nanotubes.

Figure 4 shows photographs of a SWNT bridging between fabricated cantilevers. Since the substrate below the cantilever was etched through, the SWNT was totally free-standing. It is observed that nanotubes are grown mainly on the top surface of the structures.

In order to evaluate the characteristics of the synthesized nanotubes, the Raman spectra were measured. The Spectra of nanotubes excited by a 488-nm-Ar-laser spot focused on the cantilever tip are presented in figure 5. Characteristic resonant peaks of SWNT radial breathing mode (RBM) were observed in 150-300 $\mathrm{cm}^{-1}$, which shows that SWNTs were successfully grown on the tip part of the cantilever. The high G-band/D-band ratio indicates high-quality of produced SWNTs.

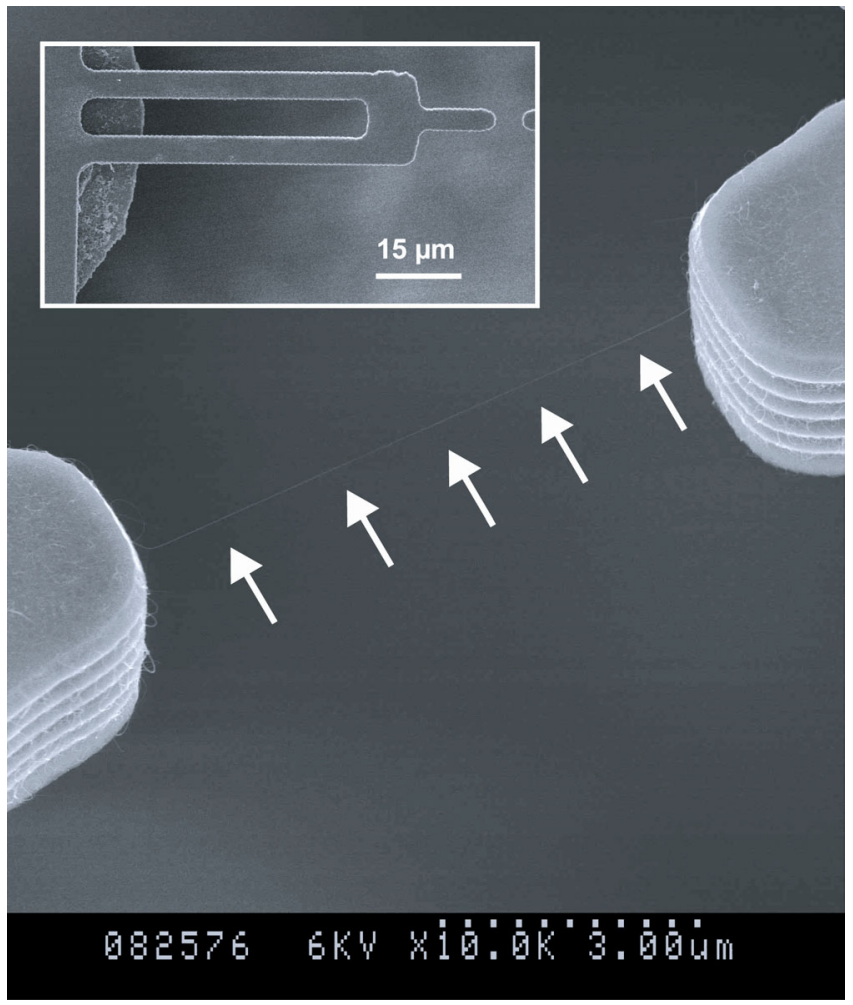

Figure 4. SEM photographs of the cantilevers and a bridging SWNT bundle synthesized by the described process. 


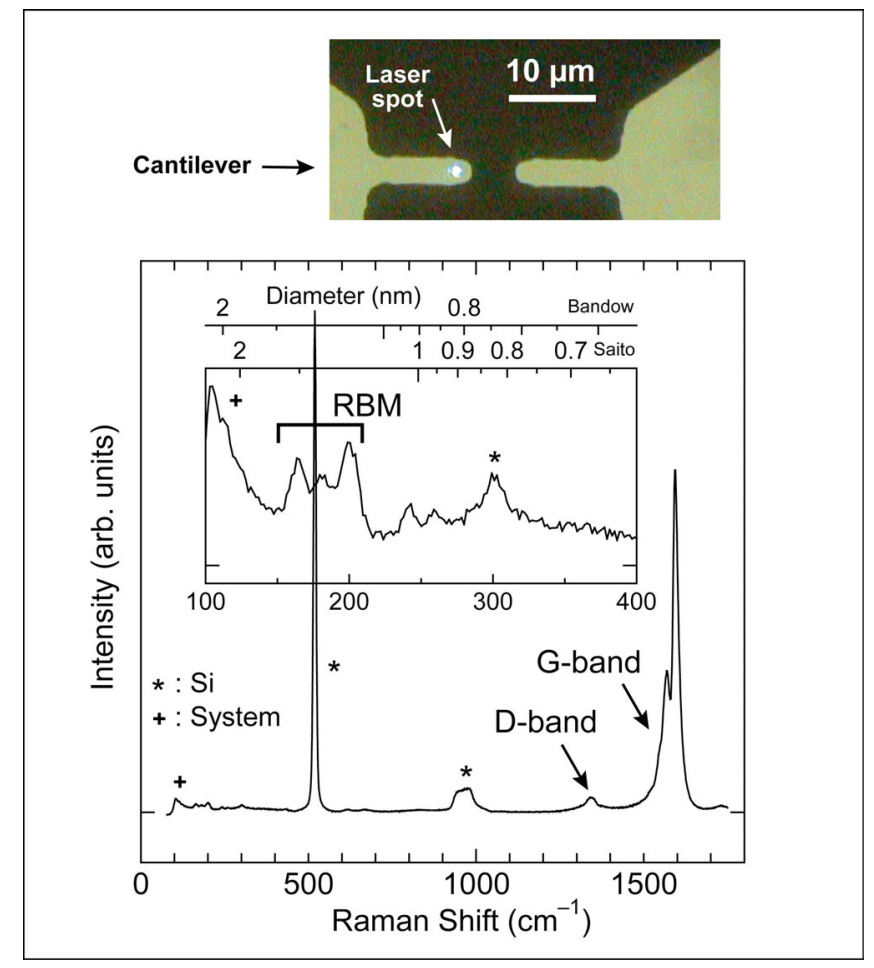

Figure 5. Raman spectrum measured by $488 \mathrm{~nm}$ excitation. Peaks measured in $150-300 \mathrm{~cm}^{-1}$ (RBM) are characteristic resonance observed in SWNTs.

\section{Cantilever Design}

According to [9], For a 605-nm-long double-side-supported SWNT bridge, $76 \mathrm{~nm}$ vertical deflection was measured with a force of $9 \mathrm{nN}$ applied at the center point. Let us assume a SWNT to be an elastic string. For the case a SWNT bridging the 5-micro-meter gap of our cantilever, vertical deflection of approximately $1.3 \mathrm{mi}-$ crometer yields the same tension, which cause the vertical force of $4.5 \mathrm{nN}$ to the cantilever. Thus the change $\Delta k$ in the spring constant $k_{c}$ of the cantilever due to a bridging nanotube becomes:

$$
\Delta k=4.5 \mathrm{nN} / 1300 \mathrm{~nm}=0.004 \mathrm{~N} / \mathrm{m} .
$$

Here are dimensions of an example cantilever:

$$
l=170 \mu \mathrm{m}, w=10 \mu \mathrm{m}, t=2 \mu \mathrm{m} .
$$

The resonant frequency and the spring constant of the cantilever are estimated to be:

$$
f=90 \mathrm{kHz}, k=0.7 \mathrm{~N} / \mathrm{m} .
$$

From (2) and (3), change $\Delta f$ in resonant frequency per one nanotube becomes:

$$
\Delta f=f \sqrt{\left(k_{c}+\Delta k_{c}\right) / k_{c}}-f=270 \mathrm{~Hz} .
$$

When the Q factor of the cantilever oscillation is assumed to be $100 \sim 10000$, the three $\mathrm{db}$ bandwidth $\Gamma$ becomes:

$$
\Gamma=9 \sim 900 \mathrm{~Hz}
$$

Since we tested various types of cantilevers with the thickness ranging from $100 \mathrm{~nm}$ to $5 \mu \mathrm{m}$ in [3], the detection of every single bridging nanotube seems relevant with the proposed configuration.

When the gap is decreased, the spring constant of a nanotube bridge becomes larger, and as a result, $\Delta f$ becomes larger. On the other hand, the number of bridging nanotubes becomes larger for smaller-gap structures (compare figure 1 and 4), and it will be more difficult to distinguish every single bridge growth. The spring constant and the Q factor of the cantilever should be chosen for each experimental CVD condition.

\section{EXPERIMENT}

Mechanical frequency response of a fabricated cantilever was measured under the process conditions (figure 6).

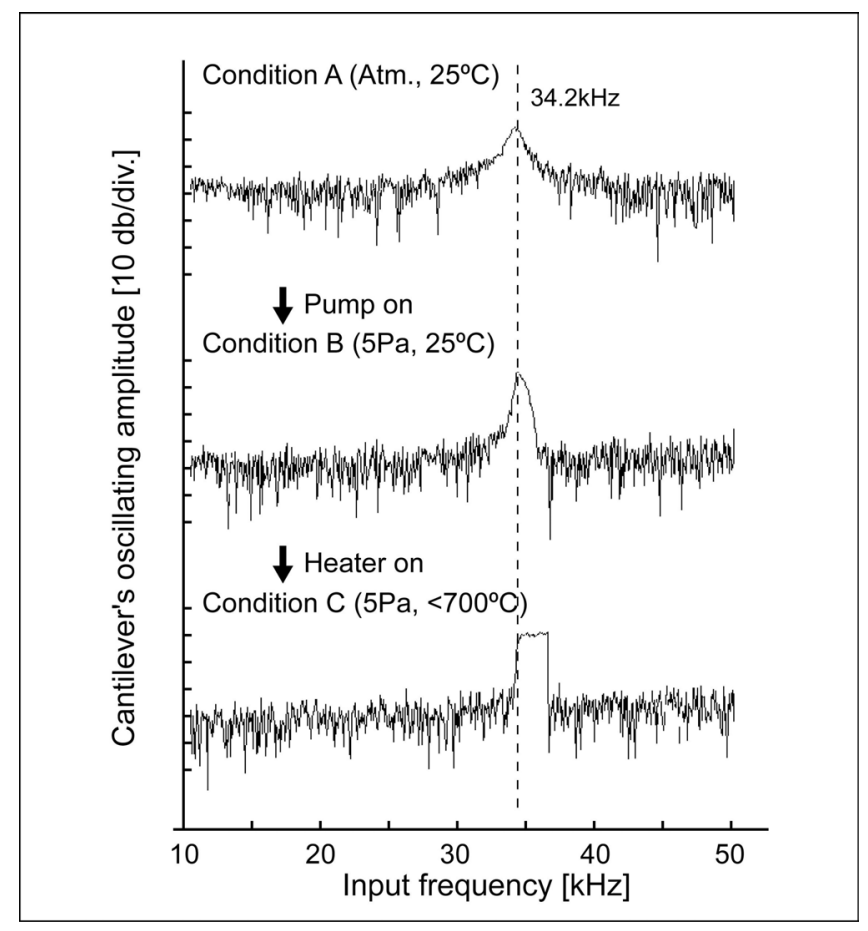

Figure 6. Mechanical frequency response of a cantilever under the low-pressure and high temperature conditions.

After the quartz tube was evacuated (condition B), a larger $\mathrm{Q}$ factor was observed. The spot-heater was then turned on condition C. A much larger oscillating amplitude was measured because the elastic modulus of $\mathrm{Si}$ changes as the cantilever was heated. In condition $\mathrm{C}$, the signal went out of the measuring range because the amplitude of the cantilever's driving voltage was set to the same as the condition 
A. Although oxidation and reduction of the structure silicon must be taken into account for resonators working at high temperatures, the change in the resonant frequency was not significant in this experiment. The inside of the reaction chamber was evacuated before and during the heating process.

\section{Conclusion}

We showed mechanical properties of silicon micro probing cantilevers for real-time in-situ measurement of SWNT growth. The cantilever oscillates under the high-temperature and low-pressure condition of the newly proposed catalytic CVD process, which successfully synthesized SWNTs on the cantilever.

The proposed configuration of the cantilever was proved to possess a capability of detecting every bridging SWNT regardless of its conductive properties. The setup can be easily combined with other measuring techniques to reveal the almost unknown opto-electro-mechanical properties, such as Raman spectra, conductivity, elasticity and etc., of SWNTs during the growing process.

\section{ACKNOWLEDGEMENTS}

This research is supported by the PROgram for the promotion of Basic Research Activities for Innovative biosciences (PROBRAIN), under the supervision of the Ministry of Agriculture and Fisheries in Japan. The photolithography masks were fabricated using the EB lithography apparatus of the VLSI Design and Education Center (VDEC) of the University of Tokyo. The Alcatel 601E ICP etching system was used to pattern the silicon structures.

\section{REFERENCES}

[1] A.M. Cassel, N.R. Franklin, T.W. Tombler, E.M. Chan, J. Han, and H. Dai, "Direct Growth of Free-standing Single-walled Carbon Nanotubes," Journal of American Chemical Society, 121, pp. 7975-7976, 1999.

[2] M.S. Dresselhaus and G. Dresselhaus, Science of Fullerenes and Carbon Nanotubes, Academic Press, 1996.

[3] M. Gel and I. Shimoyama, "Sub-Micron Thick High Sensitive Piezoresistive Cantilevers by Boron Etch Stop and Argon Implantation," proceedings of IEEE MEMS, pp. 494-497, 2003.

[4] J.H. Jeong, S.H. Chung, S.H. Lee and D. Kwon,
"Evaluation of elastic properties and temperature effects in Si thin films using an electrostatic microresonator," Journal of Microelectromechanical systems, 12: (4), pp. 524-530, 2003.

[5] S. Maruyama, R. Kojima, Y. Miyauchi, S. Chiashi, M. Kohno, "Low-temperature synthesis of high-purity single-walled carbon nanotubes from alcohol," Chemical Physics Letters 360, pp. 229-234, 2002.

[6] Y. Murakami, Y. Miyauchi, S. Chiashi, S. Maruyama, "Characterization of single-walled carbon nanotubes catalytically synthesized from alcohol," Chemical Physics Letters 374, pp.53-58, 2003.

[7] Y. Murakami, Y. Miyauchi, S. Chiashi, S. Maruyama, "Direct synthesis of high-quality single-walled carbon nanotubes on silicon and quartz substrates," Chemical Physics Letters 377, pp. 49-54, 2003.

[8] T. Ono, D. Wang, S. Sugimoto, H. Miyashita and M. Esashi, "Nanomechanics of Ultrathin Silicon Beams and Carbon Nanotubes," proceedings of IEEE MEMS, pp. 33-36, 2003.

[9] T.W. Tombler, C. Zhou, L. Alexseyev, J.K. Hongjie, L. Liu, C.S. Jayanthi, Meijie Tang and S.Y. Wu, "Reversible electromechanical characteristics of carbon nanotubes under local-probe manuplation," Nature, vol. 405, pp.769-762, 2000. 